\title{
Construction and configuration of convection-powered asphalt solar collectors for the reduction of urban temperatures
}

\author{
A. Chiarelli*, A. Al-Mohammedawi, A.R. Dawson, A. García \\ Nottingham Transportation Engineering Centre (NTEC), Faculty of Engineering, The \\ University of Nottingham, University Park, Nottingham, NG7 2RD
}

\begin{abstract}
In this paper, an analysis of a convection-powered asphalt solar collector prototype is approached by the means of experimental trials and computational fluid dynamics (CFD) simulations in order to evaluate how to optimise its design for the reduction of high urban pavement temperatures. Since the energy harvesting setup consists of a series of pipes buried in the pavement, their arrangement is here studied and experimentally compared to a possible construction technique consisting of concrete corrugations that aim at replacing the pipes. CFD simulations are employed to optimise the air collection chamber which is placed immediately before the heated air leaves the asphalt solar collector prototype. The data gathered is analysed in terms of energy harvested and exergy.

The results obtained show that for an overall optimal performance, pipes should be installed in a single row under the pavement wearing course. This allowed a surface temperature reduction of up to $5.5^{\circ} \mathrm{C}$ in the pavement prototype studied and the highest absorbed energy and exergy measured. In addition, the CFD simulations showed that care has to be put in finding the optimal shape and size for the air collection chamber, as they significantly influence the behaviour of the system.
\end{abstract}

${ }^{*}$ Corresponding author

Email addresses: chiarelli.andrea@gmail.com (A. Chiarelli),

evxab10@nottingham.ac.uk (A. Al-Mohammedawi), andrew.dawson@nottingham.ac.uk

(A.R. Dawson), alvaro.garcia@nottingham.ac.uk (A. García)

Preprint submitted to the International Journal of Thermal Sciences 
Keywords: asphalt solar collector, air convection, energy harvesting, asphalt pavement, CFD

1

4

5

\section{Introduction}

High pavement temperatures are known to be responsible for structural damages of pavements such as premature rutting [1, 2], reduced comfort for people due to overheating of buildings in an urban enviroment [3], and an increased energy consumption related to the Urban Heat Island (UHI) effect [1, 4, 5, 6, 7. These phenomena are affected by a combination of the paving materials chosen and the weather conditions present in a chosen location, thus, their likelihood is a function of pavement design and location. Due to the fact that location is not an actual variable, it appears clear that appropriate design choices are fundamental to ensure the minimisation of the damages and the discomfort that can arise from high pavement temperatures.

In summer, due to the effect of weather conditions and thermal radiation from buildings, pavement surface temperatures reach peak values, which can get as high as $70^{\circ} \mathrm{C}$ [8], therefore, techniques to lower them have been investigated. Research in this field is usually pursued by studying the effect of changes in the materials being used. Examples of the properties modified by researchers include thermal conductivity, specific heat capacity, albedo, and emissivity 9, 10, 11, 12, 13, 14, 15, 16, 17. It is also relevant to mention that asphalt pavements naturally suffer loss of colour over time due to solar radiation. As a consequence, their thermal behaviour changes without the need of any modification and usually implies a slight reduction in the pavement temperature and energy storage capacity [18].

A different approach for the reduction of surface temperatures consists in the circulation of a fluid under the pavement wearing course for the purpose of absorbing energy and, thus, reducing the pavement temperature. This can be done using water [19, 20, 21, 22, 23] or by exploiting natural convection, as done by the authors in [24, 25, 26]. The use of natural convection to power energy 

49 is usually water.

harvesting requires channels under the wearing course of roads in order to allow the generation of buoyancy-driven air flow, which is able to absorb heat from the upper layers of the pavement. The warmed-up air flowing under the pavement is expelled through a chimney, where the heat may be used for a chosen application. The influence of the chimney height and diameter and the effect of the inlet temperature in the system have been previously discussed [25, 26], however, no studies focused on the shape and arrangement of the air channels installed under the pavement or on the role of the air collection chamber, i.e., the volume where air is accumulated before exiting the pavement through the chimney, which is here abbreviated to "air box" [26].

In this paper, an asphalt solar collector prototype is experimentally studied with a number of different pipe arrangements and with a novel configuration based on concrete corrugations meant to replace pipes. The results obtained here are intended to demonstrate that a realistic technique to implement convection powered energy harvesting can be developed and that concrete corrugations are a possible candidate for this task. Furthermore, the effect of changes in the size and shape of the air box are here studied by the means of computational fluid dynamics (CFD) simulations to assess their influence on the air speed and temperature at the chimney outlet. The novelty of the present study is in the fact that convection-powered asphalt solar collectors for the purposes of pavement temperature reduction are not studied in the literature, since the operating fluid

\section{Experimental methods}

\subsection{Study of pipe arrangements}

An asphalt solar collector prototype was built with the same general structure as shown in [25] and [26]. The system (Fig. 1a] is made of two layers, i.e., an asphalt wearing course (maximum aggregate size of $10 \mathrm{~mm}, 6 \%$ air void content, $50 \mathrm{~mm}$ thickness) and an aggregate layer (silica sand, $130 \mathrm{~mm}$ thickness). A set of $6,1 \mathrm{~m}$ long, copper pipes were buried in the aggregate layer in 5 dif- 


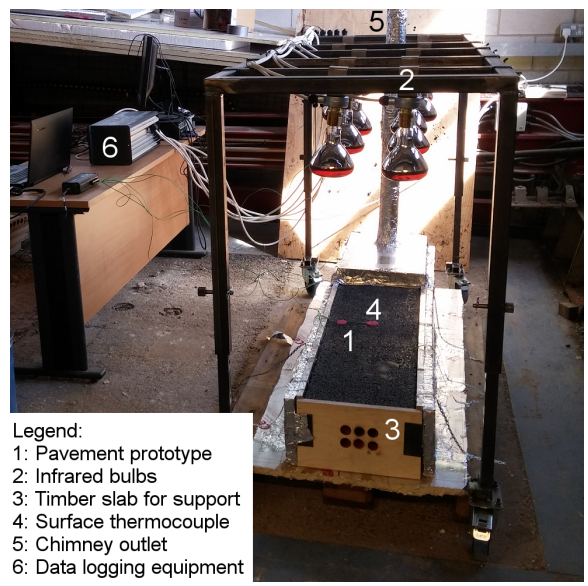

(a) Photo of the prototype in the laboratory.

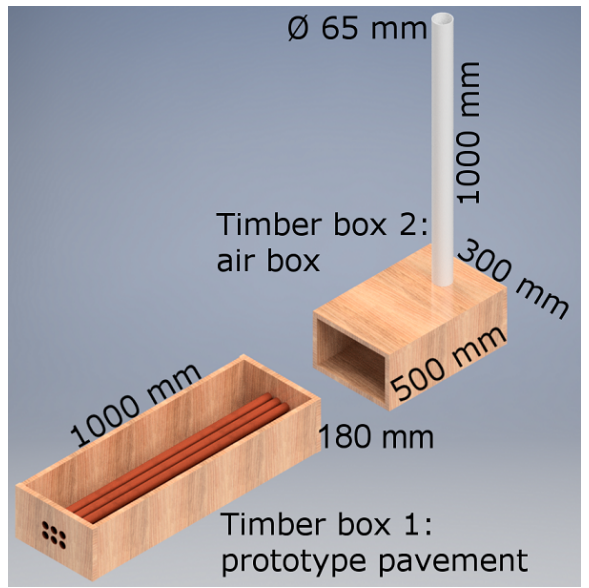

(b) Main parts of the prototype disassembled (project phase rendering).

Figure 1: Asphalt solar collector prototype powered by air convection.
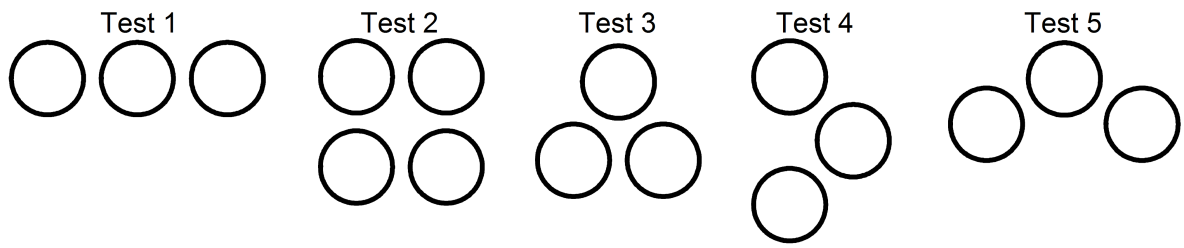

Figure 2: Configurations of the pipes in the experiments, centre-to-centre distance $37.5 \mathrm{~mm}$. 
ferent configurations (see Fig. 2). Since in the existing literature there is no guidance on the effect of pipe spacing and arrangement in convection-powered asphalt solar collectors, it was decided to compare the pavement prototype to a shell-and-tube heat exchanger and to test the pipe configurations that are generally used in such a common and widely studied component. As shown in Fig. 2, five configurations were chosen according to the design guidance provided in 27. An overview of all the tests performed is available in Tables 1 and 2 The pipes are supported by the front and back panels of the prototype, which also provide a precise control of their position and pitch ratio (center-to-center distance, $37.5 \mathrm{~mm})$. The remaining sides of the prototype were built with timber slabs (18 mm thickness) and thermally insulated with extruded polystyrene foam and bubble foil insulation so as to ensure no external heat loss.

The receptor chamber, into which the air from the pipes flows is called the air box [25, 26]. On the top of the air box, a chimney was installed to form the system outlet (see Fig. 1).

In Fig. $1 \mathrm{~b}$ the components of the prototype are displayed side-by-side in order to allow a clearer understanding of the interrelationship between the two separated timber boxes of which the system is made.

It is important to point out that the experimental method chosen for the analysis of the pipe arrangements was aimed at assessing the effectiveness of the system when the same total volume of pipes is installed in different ways. Therefore, the results obtained evaluate the energy harvesting solar collector based on this parameter and no considerations can be made based on different criteria, e.g., pipes installed per unit width of pavement. This is because to do so it would be highly important to keep into account edge effects and the influence of nearby pipes, which would have a significant influence on such kind of analysis. In this paper, since all the pipes are considered together and they are placed at a high enough distance from the sides of the prototype, edge effects are not expected to have a strong influence on the final results. 


\begin{tabular}{ll} 
Test number & Configuration of the pipes or air channels \\
\hline 1 & Pipes in a single row \\
2 & Pipes installed in two rows, superimposed layers \\
3 & Pipes installed in two offset rows with angle of $60^{\circ}$ between pipes \\
4 & Pipes installed in three offset rows with angle of $45^{\circ}$ between pipes \\
5 & Pipes installed in two offset rows with angle of $30^{\circ}$ between pipes \\
6 & No energy harvesting pipework \\
7 & Concrete triangles \\
8 & Concrete semicircles
\end{tabular}

Table 1: Overview of the experiments performed.

\begin{tabular}{ll} 
Test number & Configuration of the air box \\
\hline 10 & Air box with $1 / 2$ length and rectangular section $\left(V=15.3 \mathrm{dm}^{3}\right)$ \\
11 & Air box with $1 / 4$ length and rectangular section $\left(V=7.65 \mathrm{dm}^{3}\right)$ \\
12 & Air box with $1^{1 / 2}$ length and rectangular section $\left(V=45.9 \mathrm{dm}^{3}\right)$ \\
13 & Air box with real length and triangular section $\left(V=15.3 \mathrm{dm}^{3}\right)$ \\
14 & Air box with $1 / 2$ length and triangular section $\left(V=7.65 \mathrm{dm}^{3}\right)$ \\
15 & Air box with $1 / 4$ length and triangular section $\left(V=3.825 \mathrm{dm}^{3}\right)$ \\
16 & Air box with $1^{1 / 2}$ length and triangular section $\left(V=22.95 \mathrm{dm}^{3}\right)$ \\
17 & Manifold geometry $\left(V=0 \mathrm{dm}^{3}\right.$, see Fig. 3
\end{tabular}

Table 2: Overview of the computational simulations performed.

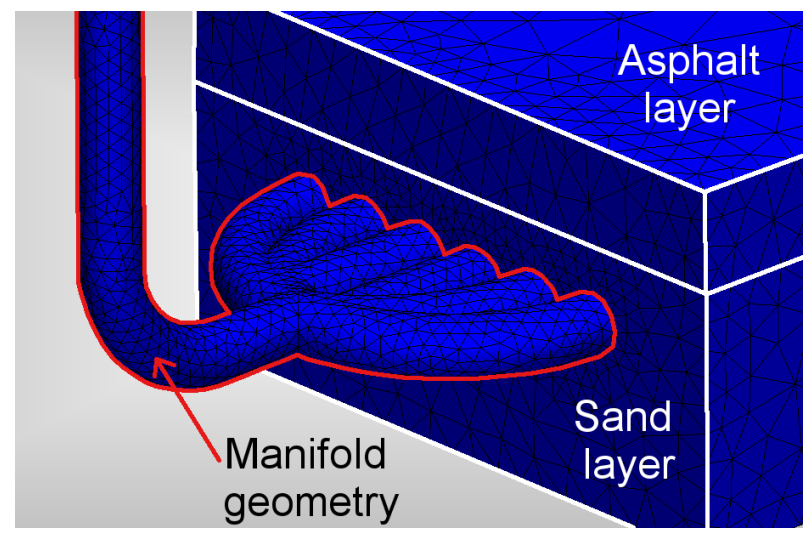

Figure 3: Manifold geometry used for Test 17. 


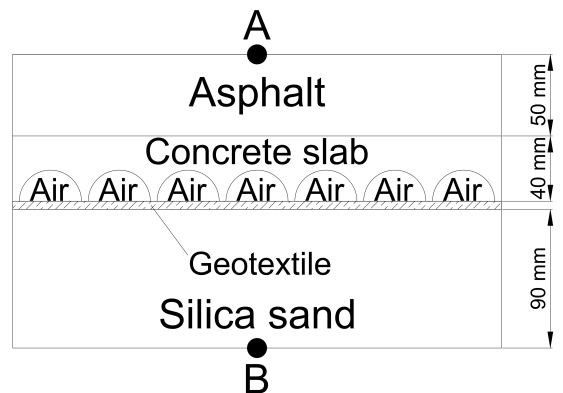

(a)

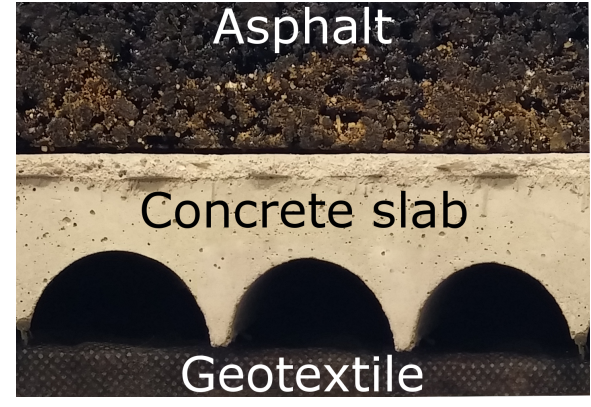

(b)

Figure 4: Experimental configuration for testing concrete corrugations in the asphalt solar collector. (a) Scheme of the concrete slabs setup and position of the thermocouples. (b) Photo of the concrete slabs installed in the prototype..

\subsection{Study of concrete corrugations as a construction technique}

In the current literature, construction techniques for the implementation of convection-powered asphalt solar collectors are not studied. For this reason it is necessary to propose a new method for the construction so that this kind of asphalt solar collectors can be considered. In particular, two $40 \mathrm{~mm}$ thick concrete slabs were cast in order to replace the pipes considered in the previous literature [24, 25, 26] and in subsection 2.1.

The shapes considered for the concrete slabs are triangles and semicircles and their size was chosen to obtain the same total volume as the pipes in order to allow a direct comparison between the two different solutions (see Fig. 7).

The concrete slabs were installed in the prototype just below the asphalt surface, thus, leaving a $90 \mathrm{~mm}$ high volume to be filled with silica sand (see Fig. 4a). As shown in Fig. 4 , between the concrete slabs and the silica sand a thin geotextile membrane was installed to prevent the roughness of sand from influencing the results.

The mix design for the concrete used to form the slabs is not discussed here, as it is not relevant for the present study because only heating and cooling properties are considered. 


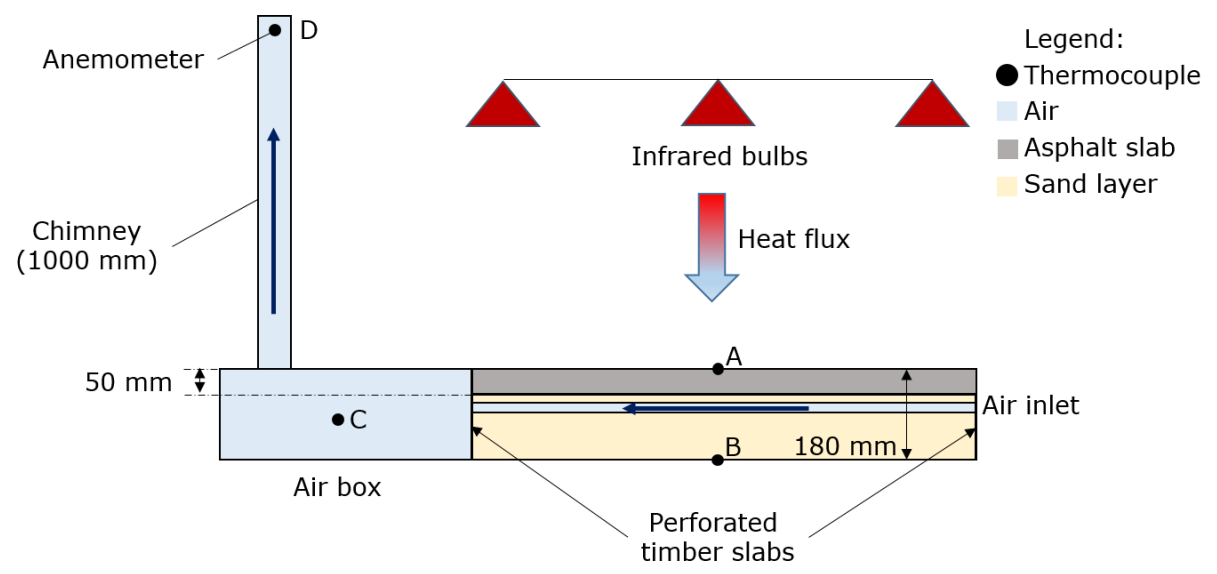

Figure 5: Cross section of the experimental setup and position of the thermocouples.

\subsection{Tools and testing conditions}

The prototype under investigation was intended to simulate the energy withdrawal from a hot pavement, therefore, a heating system was used to simulate the Sun's radiation. As shown in Fig. 1a and 5, this system consists of a steel structure holding a set of 6 infrared light bulbs that are able to take the surface temperature to $80^{\circ} \mathrm{C}\left(176^{\circ} \mathrm{F}\right)$. A high temperature was preferred to more realistic values (e.g., $70^{\circ} \mathrm{C}[8]$ ) due to the fact that previous tests showed that, with higher temperatures, it is easier to experimentally detect the differences between setups. The reason for this is related to the fact that, in a laboratory environment, there are many thermal phenomena that cannot be controlled but which may influence the results, e.g., thermal convection and radiation. Thus, by using temperatures much higher than the environmental temperature it is easier to detect differences in the results consequential upon differences in the layouts analysed.

With reference to Fig. 5 and 4 a, thermocouples were used to measure temperatures on the asphalt surface (position A), at the bottom of the system (position B), in the air box (position C), and at the chimney outlet (position D). The 
data was collected with an OMEGA OMB-82 DAQ-54 datalogger ${ }^{1}$

Finally, the air speed at the system outlet (chimney, position D) was measured with a thermal anemometer at the end of every test. All the tests were run for 24 hours to reach steady state conditions and a target surface temperature of about $80^{\circ} \mathrm{C}$.

\subsection{Theoretical background for the analysis of experimental data}

The data gathered in the various pipe configurations cannot be presented directly with any meaning because the whole system under analysis is changed every time. Therefore, the data needs to be processed to allow a meaningful comparison. In this paper, this is done by calculating the energy absorbed in the energy harvesting experiments performed in the laboratory. Note that, from this point onwards, all equations use SI units.

The energy absorbed by the operating fluid, $q_{a b s}$, can be quantified as explained in [26]:

$$
q_{a b s}=\dot{m} \cdot c_{p} \cdot\left(T_{c}-T_{e}\right)
$$

where $\dot{m}$ is the mass flow of air in the chimney, $c_{p}$ is the specific heat capacity, $T_{c}$ is the temperature of air at the chimney outlet, and $T_{e}$ is the temperature of environmental air. The value of heat flux obtained is an approximation, as it assumes that the air velocity through the system, the density, and the specific heat capacity are constant. This is not completely accurate, as in the air box there are eddies due to the change of direction of the flow and because density changes due to air warming up. For a visual confirmation of the presence of eddies in the air box, it is sufficient to represent particle traces when performing CFD simulations of the energy harvesting setup.

The mass flow of air is here calculated as:

$$
\dot{m}=\rho \cdot v \cdot A
$$

\footnotetext{
${ }^{1}$ For more information, see http://www.omega.co.uk/pptst/OMB-DAQ55.html.
} 
where $\rho$ is the density of air, $v$ is the speed at the chimney outlet, and $A$ is the cross section of the chimney. The density used in Eq. 2 is calculated with the ideal gas law:

$$
\rho=\frac{p_{a}}{R \cdot T_{c}}
$$

where $p_{a}$ is the atmospheric pressure $(101325 \mathrm{~Pa}), R$ is the specific gas constant for dry air, $287.058 \mathrm{~J} /(\mathrm{kg} \mathrm{K})$, and $T_{c}$ is the temperature at the chimney outlet. Finally, in order to provide further insight for the interpretation of the results, it is interesting to introduce the use of exergy in the place of energy [28, 29]. The calculation of exergy allows a more realistic representation of the energy that is available after the harvesting process because it considers the temperature of the environment and the temperature of the heat source through the use of the Carnot factor to convert the thermal energy into work [30]. For this reason, exergy can be defined as the maximum amount of work that can be obtained from the harvested energy [29] and allows a more functional comparison between the pipe arrangements under analysis. The Carnot factor, $\eta_{t h}$, can be calculated as :

$$
\eta_{t h}=1-\frac{T_{e}}{T_{c}}
$$

where $T_{e}$ is the temperature of the environment and $T_{c}$ is the temperature of the air at the chimney outlet, (both in degrees K). From Eq. 4 it appears clear that the closer the temperature at the chimney outlet is to the environmental temperature, the lower the value of the Carnot factor will be. The Carnot factor can be used to calculate the exergy associated with the heat absorbed by the operating fluid, $B_{a b s}$ :

$$
B_{a b s}=\eta_{t h} \cdot q_{a b s}
$$

Therefore, a low value of the Carnot factor will yield a low value of exergy, meaning that the configuration being considered provides heat at a temperature that is too close to the environmental temperature to be used effectively and efficiently in a thermal device.

In this paper, the calculation of the exergy is used as a means to objectively compare the different pipe arrangements considered. The reason for this is the 
need to find a physical parameter that can be calculated for all the scenarios studied and that is independent of the specific geometric configuration used. In fact, the performance of the experimental setups used in this paper differs due to a combination of geometry, heat transfer, and fluid-dynamics, therefore, the scenarios cannot be effectively compared based on a single criterion such as the outlet air speed or the temperature reduction they allow.

\section{Computational methods}

Due to the simplicity of the experimental setup, CFD simulations are an appropriate tool to evaluate computationally changes in its shape and size (see, e.g., 31]). The analysis is carried out by combining the first law of thermodynamics, the Navier-Stokes equation, and the principle of mass conservation in a three-dimensional representation of the prototype pavement. The simulations are performed in steady state conditions.

The geometry considered for the prototype pavement is the simplest one under analysis, i.e., the configuration corresponding to Test 1 (pipes in a single row). This choice is arbitrary and simulations involving variations in the design of the air box with different pipe arrangements may yield different numerical results. However, it can be hypothesised that such differences would be only in the numerical values of the temperatures obtained and not in the trends of the results due to the fact that the volume of air contained in the pipes is significantly lower than the volume contained in the air box. As a result, the effects of a variation in the design of the air box are expected to be higher than those caused by changes in the design of the pipe arrangements.

For the purposes of a validation of the results obtained in the simulations, these are here briefly compared to the values obtained experimentally. Should the prototype under analysis be installed in the natural environment, the CFD results would benefit from a further validation such as that developed in [19]. However, due to the simplicity of the system and the fixed boundary conditions described in the next section, this was not pursued in the present article. 
Furthermore, fluid dynamics simulations are here used to compare the two concrete corrugations considered. Simplified flow simulations were run in single channels with the appropriate cross section (see Fig. 7) and with a chosen pressure difference $(20 \mathrm{~Pa})$ between inlet and outlet. By doing this it was possible to compare the outlet air speeds obtained with the two configurations and draw conclusions about how friction losses related to the shape of the channels affected the performance. The use of $20 \mathrm{~Pa}$ as the pressure difference is motivated by the fact that values in this order of magnitude are commonly used to achieve a controlled natural convective flow between different areas of a building [32, 33, thus, they are expected to be representative for a small air mass flow such as that found in the experimental part of the present investigation. The pressure difference chosen is arbitrary, however, the results of the simplified simulations are only used to compare the head losses in the different concrete corrugations and are not meant to be representative of the real mass flows and velocities that were measured in the experimental phase.

The same kind of analysis could not be used to compare the results of Tests 1-5 because they share the same cross section shape while the pipe arrangement changes, thus, the difference in their performances is not expected to be related only to different head losses caused by the shape of the channels. For this reason, a computational reproduction of these tests would not be helpful for the interpretation of the results, as it would simply reproduce what was seen experimentally.

\subsection{Study of the size and shape of the air box}

In a series of preliminary computational simulations performed by the authors, the size and shape of the air box volume were found to highly affect the movement of air from the pipes to the chimney outlet. Therefore, a parametric analysis of the air box volume was performed with the software Autodesk ${ }^{\circledR}$ CFD to assess the extent of such influence. The analysis of the air box volume, $V$, was based on the variation of its length and section, thus, keeping the rest 


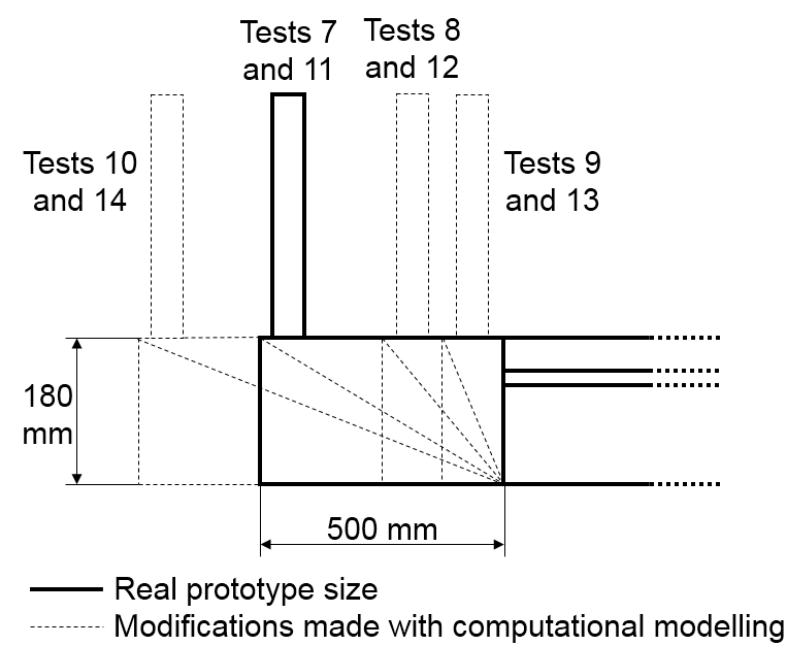

Figure 6: Graphical explanation of the modifications to the air box considered in the CFD simulations.

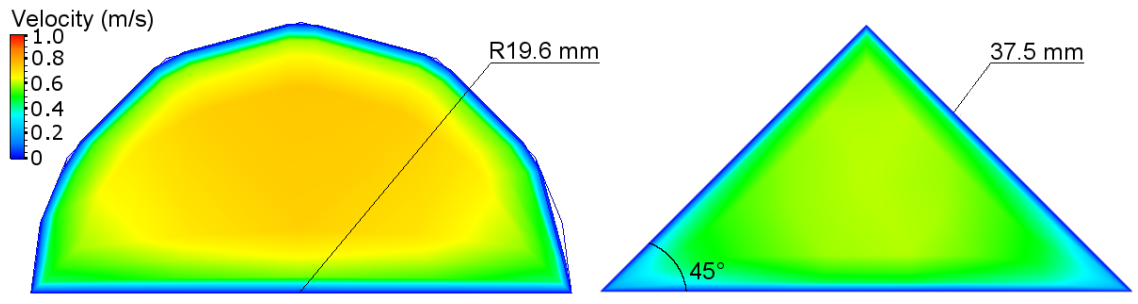

Figure 7: Semicircular and triangular cross sections used in the computational study. 
of the prototype as is. The CFD simulations performed considered the configurations listed in Table 2 A graphical explanation of the configurations analysed (Tests 9-17) is available in Fig. 6. The boundary conditions used for the CFD simulations are the environmental temperature $\left(21^{\circ} \mathrm{C}\right)$ at the system inlet, environmental pressure at the chimney outlet (set as a null gauge pressure), and a pavement surface temperature of $70^{\circ} \mathrm{C}[8$. Since the boundaries of the system are held at constant values of the relevant parameters, the boundary conditions can be classified as Dirichlet conditions.

In this paper, thermal convection and radiation on the pavement surface are not simulated, as they would need further hypotheses such as the choice of the speed and temperature of the air flowing above the pavement. For this reason, there is no need to use a higher surface temperature as done for the experiments in the laboratory to compare the different layouts of the system.

The air speeds and temperatures at the chimney outlet obtained for each case are compared and the best performing configuration(s) identified.

Finally, it is important to mention that by setting a surface temperature as a boundary condition the various configurations cannot be compared based on their effectiveness in the reduction of the surface temperature. This can be easily achieved by setting a surface heat flux as the boundary condition. In this paper, the focus of the computational study is on the role of the air box in the performance of the system, therefore, the best way to compare the different sizes and shapes under investigation is to have a common surface temperature in all the simulations. In fact, setting a heat flux would result in different surface temperatures caused by the difference in the air box sizes and shapes, which would not allow a comparison based on a common criterion (i.e., the same surface temperature). 


\section{Results}

\subsection{Experimental results}

The results gathered in the experiments are shown in Fig. 8, 10. For the figures, the energy harvested shown on the vertical axis was calculated for steady state conditions with Eq. 1 considering a hypothetical period of $1 \mathrm{~h}$.

In Fig. 8 and Fig. 9 a temperature difference is shown on the horizontal axis. This temperature difference represents the effect provided by the energy harvesting asphalt solar collector compared with a scenario with no energy harvesting. In particular, $T_{s}$ is the surface temperature, $T_{b}$ is the bottom temperature, and the subscript $N H$ means "No Harvesting".

It important not to look for trends when observing the data in Fig. 8 , Fig. 9 and Fig. 10, because the points belong to different datasets and, therefore, are represented together only for comparison purposes. The data in Fig. 8, 10 can be used to find out which configuration yields the best performance based on a chosen design objective.

A preliminary look at the data shown in Fig. 8 and Fig. 9 suggest that the installation of all pipes in a row is a very effective option, as it provides the highest surface temperature reduction, the highest harvested energy, and a high air speed. In addition, it is interesting to point out that the novel application with concrete corrugations presented in this paper managed to reach the same air speed as Test 1 (Pipes in a single row), even if the temperature reduction effect was not as noticeable.

Finally, it is important to analyse the values of energy and the values of exergy obtained as explained in Section 2.4. The relative position of the points represented in Fig. 8 10 for the energy and the exergy does not change, however, the range of variation for the two physical quantities is very different. In particular, the points representing the harvested energy range between about $60 \mathrm{~kJ}$ and $100 \mathrm{~kJ}$, while their exergy ranges between $20 \mathrm{~kJ}$ and $40 \mathrm{~kJ}$. Thus, the relative difference between the best and worst performing scenarios in terms of exergy is as high as $50 \%$, compared to a maximum relative difference of $40 \%$ when energy 

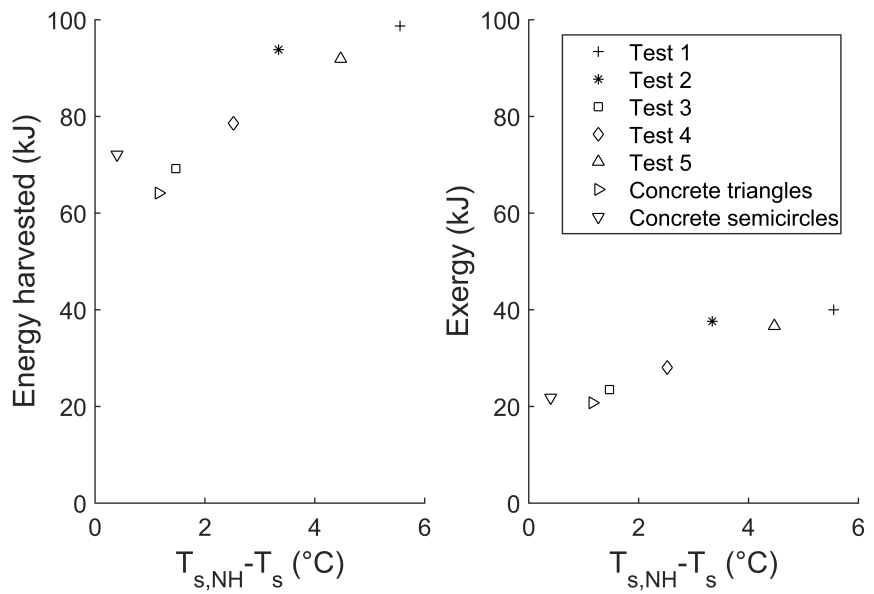

Figure 8: Surface temperature difference with no harvesting vs. Energy harvested and exergy.

288 is considered. 

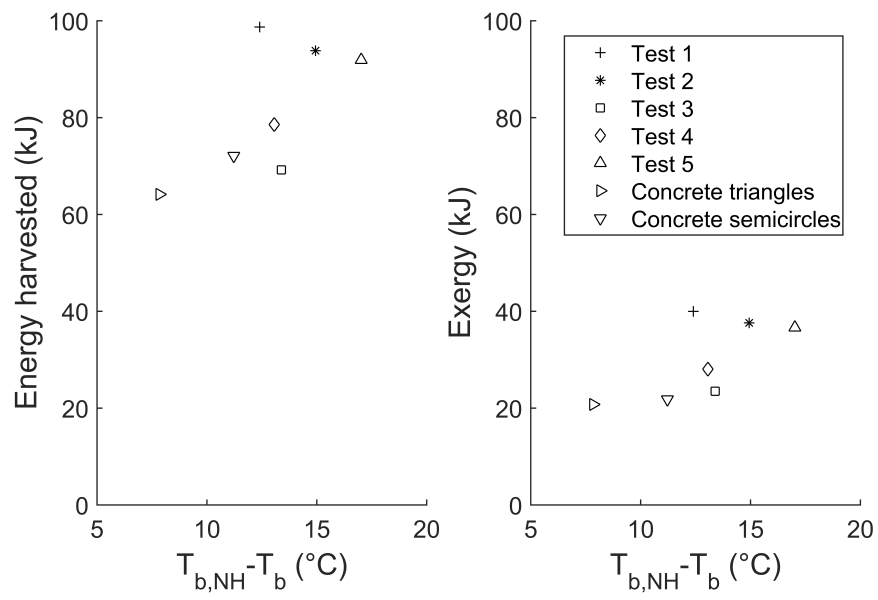

Figure 9: Bottom temperature difference with no harvesting vs. Energy harvested and exergy.
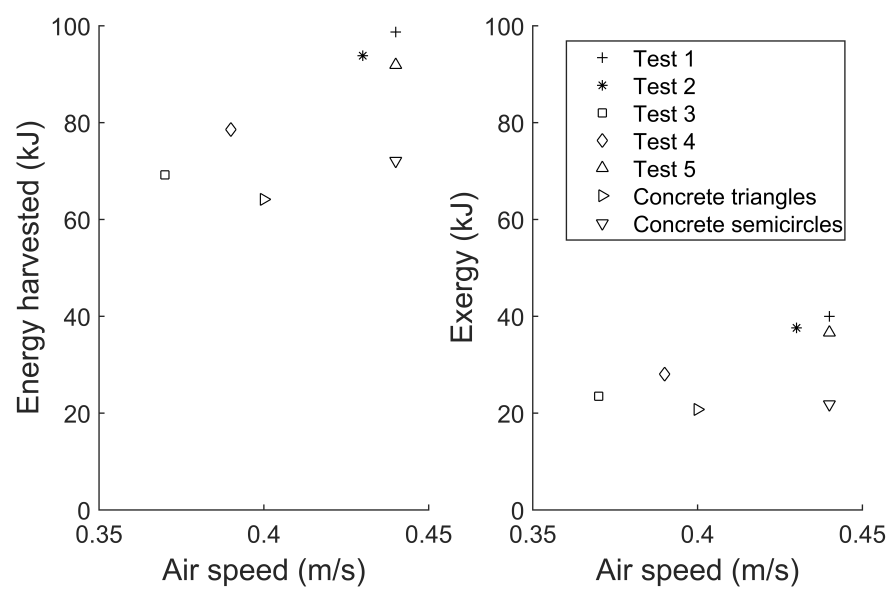

Figure 10: Air speed vs. Energy harvested and exergy. 


\subsection{Computational results}

The results of the CFD simulations run with the geometric configurations described in Section 3.1 are shown in Fig. 11, 13 as interpolated curves based on the computational results.

In Fig. 11, the temperatures of the asphalt, of the sand, of the air in the air box, and of the air at the outlet are presented for the tests where the air box has a rectangular cross section. The temperatures presented are volume-weighted averages for the subdomain they refer to. It can be seen that with an increase in the air box volume all the temperatures measured increase, except for the asphalt temperature, which has a different behaviour. In addition, the air box temperature and the outlet temperature become almost equal when the air box size increases.

In the case of an air box with a triangular shape longitudinally (i.e., the base slopes up from the heat exchanger outlet towards the chimney, Fig. 12, a very similar behaviour is seen. As for the previous case, all the temperatures in the domain except for the asphalt average temperature increase with the air box volume. The asphalt average temperature has a peak for an intermediate value of the air box volume, while it is decreasing towards the smallest and highest values of air box volume considered.

The behaviour of the air speed is shown in Fig. 13, where it can be observed that the curves peak at air speeds of approximately $0.435 \mathrm{~m} / \mathrm{s}$ and $0.43 \mathrm{~m} / \mathrm{s}$ for a rectangular and a triangular cross section of the air box, respectively.

Finally, in the case of the use of a manifold (Test 17) in the place of the air box, an average asphalt temperature of $68.8^{\circ} \mathrm{C}$, an average sand temperature of $62.2^{\circ} \mathrm{C}$, an outlet temperature of $69.2^{\circ} \mathrm{C}$, and an outlet air speed of 0.32 $\mathrm{m} / \mathrm{s}$ were obtained. As a result, it can be concluded that with a manifold the performance of the system in terms of velocity is comparable to the tests with larger air boxes, while the temperatures obtained are higher, especially in the case of the sand layer.

Furthermore, CFD simulations (see Fig. 7) were used to find the reason for the different performances yielded by the two concrete slabs (see Fig. 8 10). The 


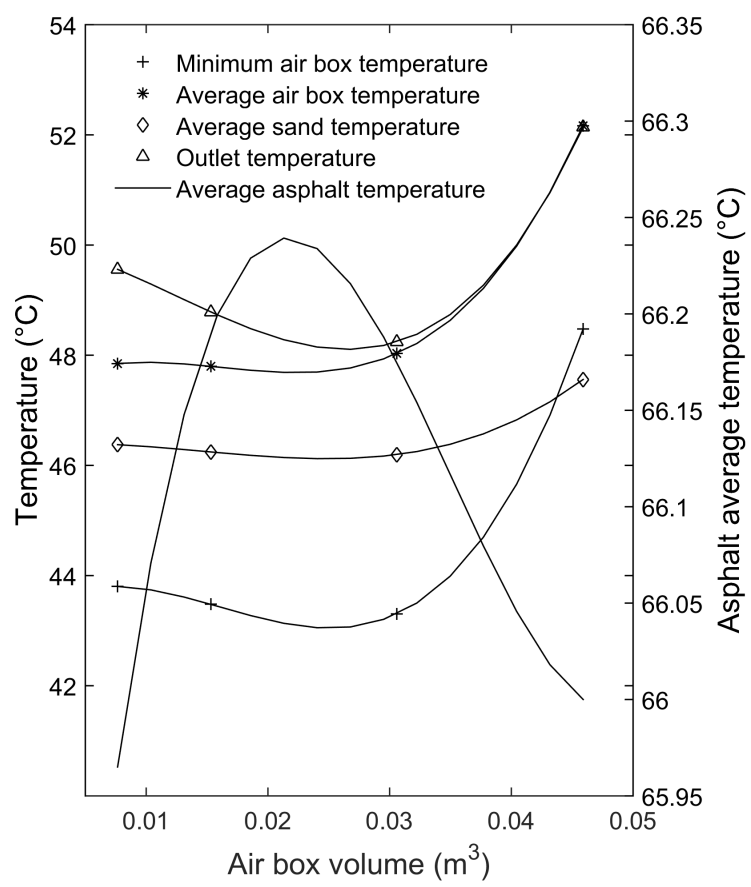

Figure 11: Effects of the air box size (rectangular cross section) on the temperatures of the components of the pavement prototype.

simulations run showed clearly that the reason why the triangular corrugations cause a lower outlet speed is that their shape generates more friction against the air flow. In particular, with a pressure difference of $20 \mathrm{~Pa}$ between inlet and outlet a relative difference in the outlet air speeds of $16 \%$ can be found between channels with a semicircular and a triangular cross section.

\section{Discussion}

\subsection{Experimental results}

\subsubsection{Energy harvested and temperature reduction effect}

The experimental results presented in this paper allow a comparison between a number of pipe arrangements in an asphalt solar collector prototype. It is im- 


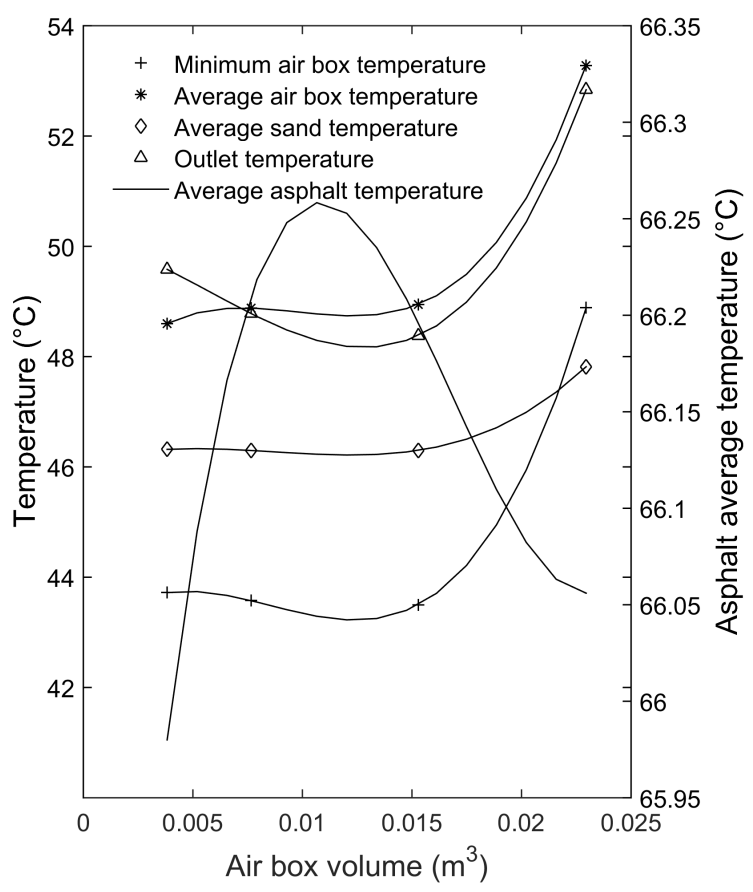

Figure 12: Effects of the air box size (triangular cross section) on the temperatures of the components of the pavement prototype. 


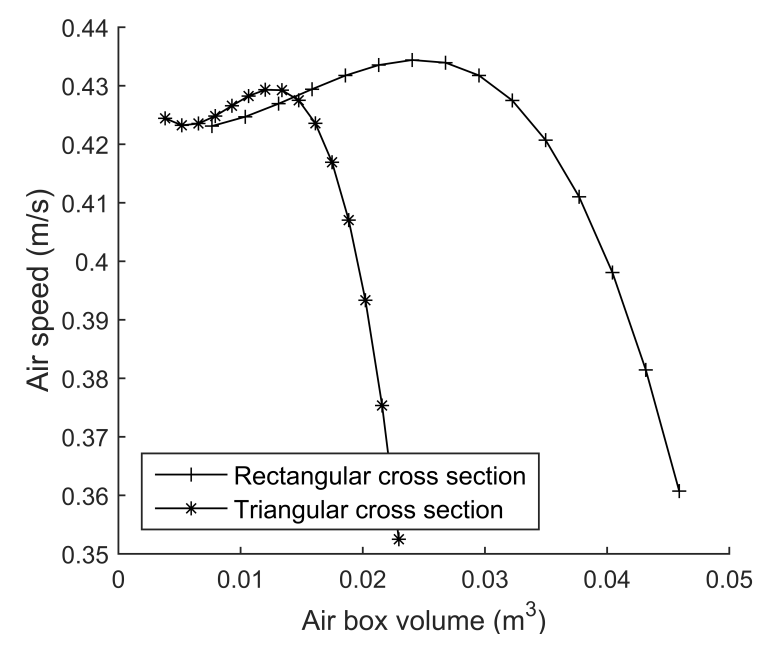

Figure 13: Outlet air speed obtained in the CFD simulations.

portant to keep in mind that the values obtained refer to a surface temperature that is higher than real surface temperatures, thus, the results represented are meant to be used for comparison purposes only. As mentioned in Section 4.1. a quick look at the results on the harvested energy suggests that quite a wide difference exists between the configurations considered and that the best performing pipe arrangement is that with all pipes in a row (see Fig. 8 10. In fact, this configuration has the highest harvested energy and the highest surface temperature reduction, a mid-range bottom temperature reduction, and the maximum outlet air speed measured. This can be considered as an overall optimal configuration due to the fact that the surface temperature reduction must be taken as of more significance than the bottom temperature because it is responsible for thermal radiation, urban overheating, the increase of the UHI effect, and surface pavement shear under trafficking. The reason for the better performance of Test 1 is simply that the pipes are closer to the pavement surface, thus, the air flowing through them is able to absorb a higher amount of heat. Other configurations are able to better control the temperature at higher depths, however, this aspect has a lower priority when the above-mentioned 
purposes are considered.

When the values of energy are compared to the corresponding values of exergy, it is clear that the actual gap between the configurations considered is not as high as it would appear when considering the values of harvested energy. In fact, when the available energy, i.e., the exergy is considered, the scenarios under analysis are converted into work and therefore can be more precisely compared based on the actual use that can be made of them. The conclusion that can be drawn from this rather small gap is that changes in the pipe arrangement only partially influence the effectiveness of the asphalt solar collector. Nevertheless, installing all pipes in a row can be confirmed as the most efficient solution for the chosen total volume of pipes.

If the concrete corrugations are considered, the experimental results show that their study should be further pursued due to the reduced pavement cooling effect. The reduced effectiveness, however, was expected due to (i) the roughness of the concrete corrugations, which increases friction in the air channels and lowers the effectiveness of heat transfer due to a reduction in the air speed; and (ii) to the higher specific heat capacity of concrete compared to that of sand, which allows sand to cool down more quickly due to the lower amount of heat accumulated in the material. In fact, this result is meant to be a proof of concept to show that the use of more realistic channels is possible and delivers a measurable result, even if small. Therefore, further studies on concrete corrugations for energy harvesting should be performed to find a more efficient construction method and an improved material.

The final remark that can be added from the analysis of the experimental data is that the energy harvested with the pavement prototype is available at a low temperature, thus, it is not very valuable in terms of work (i.e., exergy). For this reason, when uses for this energy are investigated, low-enthalpy systems such as air-source heat pumps [34] should be considered. 


\subsubsection{Theoretical considerations on the outlet air speed}

It is important to highlight that, generally, the experimental values of air speed obtained were rather low (see Fig. 10). Therefore, in the case that the air speed is relevant for the chosen application, e.g., for the use of air in a heat exchanger, some solutions can be hypothesised to solve this issue.

The simplest solution is the improvement of the thermal properties of the materials used, so that the air could absorb more energy and consequently reach a higher temperature. As a result, the pressure (and density) difference between the air box and the environment would be higher, thus, yielding a higher speed at the chimney outlet. The modification of the thermal properties of asphalt pavements has been considered in the literature [9, 10, 11, 12, 13, 14, 15, 16, 17 ] and is certainly a technically suitable solution, however, its inevitably higher cost compared to standard mixtures might reduce the likelihood of its application.

Another way to have the air absorb more heat is to increase the length of its path under the pavement, e.g., using a serpentine layout. If the path available for air flow was made more complex, however, the values of air speed may further drop rather than increase due to the fact that the motion of air is powered by natural convection and not by a fixed pressure differential (obtained, e.g., using a fan).

To better understand this it is helpful to introduce the concept of geometric tortuosity. Geometric tortuosity for porous materials is defined as the ratio between the length of a path completely inside the pores between two opposite faces of the material and the Euclidean distance between its start and end points 35]:

$$
\tau_{g}=\frac{L_{p a t h}}{L_{E}}
$$

where $\tau_{g}$ is the geometric tortuosity, $L_{\text {path }}$ is the actual length of the path in the air pores of the material, and $L_{E}$ is the Euclidean distance. Now, if a straight channel (e.g., pipes or corrugates slabs) is used to represent a highly idealised pore, its tortuosity will be equal to 1. If a more complex pipe, e.g., 
a serpentine pipe, is used, the tortuosity will increase based on the length of the path according to Eq. 6. As a result, the permeability of the air channel would decrease as the tortuosity increases. In particular, permeability to gases is usually measured using Darcy's law and is a function of the pressure difference between the ends of the material [36]:

$$
\kappa=v \frac{\mu \Delta x}{\Delta P}
$$

where $\kappa$ is the permeability of the material, $v$ is the fluid velocity in the pores, $\mu$ is the dynamic viscosity of the fluid, $\Delta x$ is the thickness of the material, and $\Delta P$ is the pressure difference between the inlet and outlet of the pore $(\mathrm{s})$. The effect of an increased tortuosity on Eq. 7 is an increased pressure needed to obtain the same value of air flow seen with, e.g., a straight pipe. This is because generally speaking more tortuous paths imply higher head losses. In fact, in a longer path the higher length will cause higher losses due to friction, while the presence of curves and possible changes in the cross section will cause localised losses related to the disruption of the flow. Since natural convection is powered by rather low pressure differences, the experimental results suggest that paths more complex than a straight line may cause the air speed to drop to too low values. The use of a serpentine would be more likely to be possible if coupled with the installation of a fan with a low energy consumption and sized to overcome all the head losses that may exist through the path, thus, obtaining an acceptable (or, potentially, better) performance. In this case, however, a thermoeconomic analysis should be performed to assess if such a setup would provide a performance that is good enough to justify the use of the resources that would be needed.

A very extreme case of a serpentine layout would be the use of the natural pores of asphalt as the air channels, thus, removing the pipes. This has been considered for water-powered energy harvesting [8] and the results obtained showed that with relatively high hydraulic gradients only low water mass flows could be obtained. In the case of natural convection of air and no electrical devices to overcome pressure losses, the situation would be even worse, because the high 
tortuosity and low permeability would cause the buoyant mass flow to be very slow and, possibly, not measurable. In addition, heated air would tend to flow upwards due to buoyancy, therefore, also the vertical permeability (perpendicular to the road surface, [37]) would be likely to become a concern. Issues related to the vertical permeability may be overcome by installing a porous asphalt layer used as an air channel between dense layers used to seal it as done in [8], however, the use of air in the place of water might require different technical solutions.

In order to verify the validity of these theoretical observations further experimental and numerical studies should be performed (i) to find the maximum tortuosity of a serpentine layout with no electrical devices that allows a natural convective air flow, (ii) to assess if there exists any combination of tortuosity of granular material pores and surface temperature that allows natural air convection, and (iii) to determine if the vertical permeability is an actual concern for buoyancy-powered flows in porous media.

\subsection{Computational results}

From a comparison between Fig. 10 and Fig. 13 it can be observed that the speed obtained computationally with the original air box volume is very close to the experimental value measured in Test $1(0.435 \mathrm{~m} / \mathrm{s}$ for the CFD simulation compared to $0.44 \mathrm{~m} / \mathrm{s}$ for the experimental value). Thus, the CFD simulation provided a realistic estimation of the physics in the system. In fact, the boundary condition set in the simulations is $70^{\circ} \mathrm{C}$, while the steady state surface temperature obtained in the experiments is $74.5^{\circ} \mathrm{C}$. For this reason, the simulated air speed is expected to be slightly lower than the experimental value obtained due to the lower energy available in the former.

The first important result found with the computational simulations is that the air box has a fundamental role in the design of the system. When a manifold geometry is used, the chimney outlet temperature is the highest found, however, as mentioned in Section 4.2, a very small temperature reduction is achieved. Therefore, the only use of the system would be related to the possible use of the 
heated air exiting the chimney, which removes one of the main purposes of the asphalt solar collector designed, i.e., achieving a temperature reduction at the pavement surface.

When an air box is used, the observation of Fig. 13 suggests that the maximum air speeds are obtained for air box volumes in the intervals $0.025-0.030 \mathrm{~m}^{3}$ and $0.01-0.015 \mathrm{~m}^{3}$ for a rectangular and a triangular cross section, respectively. If these intervals are considered in Fig. 11 and 12 it can be seen that the air box temperature, the sand temperature, and the outlet temperature are at their minimum values. On the other hand, the average asphalt temperature is approximately at its maximum point for both the cross sections considered. Therefore, when the above-mentioned intervals are considered, the thermal energy of the outlet air flow is the lowest due to the fact that the outlet temperature curves in Fig. 11 and 12 are at their minimum points.

A different way to look at the data in Fig. 11, 13 is to make a decision based on the highest outlet temperature that can be achieved, which could be useful in the case of the use of a heat exchanger for a chosen application. For both the cross sections considered, the outlet temperature is maximum at the maximum air box volume considered. In addition it appears that the curves representing the outlet temperature in Fig. 11 and 12 have an increasing trend on the right side of the interval considered. Thus, it is likely that even higher volumes would lead to higher outlet temperatures. This, however, would cause the air speed to keep decreasing, as suggested by the observation of Fig. 13 .

The conclusion that can be drawn from the analysis of the computational results obtained is that the design of the air box must be based on the effect that needs to be achieved. If this is a high outlet temperature, high air box volumes are recommended, while if a high air speed is required, the volume intervals mentioned above should be used. Furthermore, the curve of the air speed for a rectangular section has higher values for a larger interval of air box volumes, thus, its design allows more flexibility compared to that of a triangular section, where the highest speeds cover a smaller volume interval. It must be reminded that the air box design in a real life application would also depend on the fea- 
tures of the location where the harvesting pavement is installed. In fact, a larger area would probably be available based on whether the system is installed in an urban environment or not.

Finally, the CFD simulations used to compare the concrete corrugations provided an interesting explanation of the experimental results obtained with this new construction technique. In fact, since a lower outlet air speed was found with the simulation of triangular corrugations it is possible to develop a further understanding of the results seen in Fig. 8,10. The relative difference between the air speeds found experimentally for the concrete corrugations is about $10 \%$, which is in the same order of magnitude as the relative difference found between those in simplified simulations (see Section 4.2. The computational and experimental results are in agreement and their mismatch is related to (i) the fact that the pressure difference used in the simplified simulations was not the same as the experimental one (this information was not available for the physical experiments), (ii) the eddies in the air box, whose presence was not considered in the CFD analysis of the concrete corrugations for simplicity purposes even if it does influence the outlet speed, and (iii) the neglection of thermodynamics in the computational analysis of the corrugations. A lower air speed in the channels was correlated to a lower energy harvesting potential in [1], thus, the reason why the triangular corrugations were outperformed by the semicircular ones appears clear. In fact, since the semicircular corrugations cause lower fluid dynamic losses due to friction the fluid is able to reach a higher speed and, therefore, to absorb more energy from the pavement. This can be seen in Fig. $8,10$. where the absorbed energy/exergy for the triangular corrugations is lower than that found for the circular corrugations. Therefore, the use of semicircular air channels should be preferred due to their better fluid-dynamic behaviour, which in turn yields a better pavement cooling performance. 


\section{Conclusions}

In this paper, an experimental and computational analysis of the design of convection-powered asphalt solar collectors was presented. The research performed led to the following conclusions:

- The temperature reduction effect of convection-powered asphalt solar collectors found in the literature was confirmed.

- The pipe arrangement that yields the lowest surface temperature along with the highest air speed is the installation of all the energy harvesting pipes in a single row under the pavement wearing course.

- It is possible to replace pipes with concrete corrugations and obtain a pavement cooling effect. This, however, provides a reduced cooling (i.e., energy harvesting) performance, thus, further studies are encouraged.

- The fluid dynamic losses in concrete corrugations have a clear influence on the results due to the fact that a rather low pressure difference exists between the inlet and the outlet of the system. For this reason, semicircular (or circular) corrugations should be used.

- The difference between the various configurations in terms of exergy is rather low, thus, the effect of the pipe arrangements on the system performance is small.

- The exergy of the heat fluxes obtained in the experiments is low, therefore, low-enthalpy systems should be considered for possible applications using the energy harvested (e.g., using heat pumps).

- The role of the air box was clarified and it was shown that it is a fundamental part of the energy harvesting system. The use of a rectangular cross section allows flexibility, as the outlet speed is high for a wide range of air box volumes. 
- The volume of the air box needs to be chosen in the design stage based on the effect that needs to be achieved (energy generation or temperature reduction).

- The use of a manifold in the place of an air box allows a high outlet temperature, however, a very small cooling effect is achieved.

\section{Acknowledgments}

The authors thank the University of Nottingham for the financial support provided for the doctoral programme of Andrea Chiarelli.

\section{References}

[1] V. Bobes-Jesus, P. Pascual-Muñoz, D. Castro-Fresno, J. RodriguezHernandez, Asphalt solar collectors: A literature review, Applied Energy 102 (2013) 962-970. doi:10.1016/j.apenergy.2012.08.050

[2] A. K. Apeagyei, E. V. Dave, W. G. Buttlar, Effect of cooling rate on thermal cracking of asphalt concrete pavements, Journal of the Association of Asphalt Paving Technologists 77 (2008) 709-738.

[3] ANSI/ASHRAE, Standard 55-2013 - Thermal Environmental Conditions for Human Occupancy, ASHRAE, 2013.

[4] J. S. Golden, K. E. Kaloush, Mesoscale and microscale evaluation of surface pavement impacts on the urban heat island effects, International Journal of Pavement Engineering 7 (2006) 37-52. doi:10.1080/10298430500505325

[5] O. S. Pinho, M. D. Manso Orgaz, The urban heat island in a small city in coastal Portugal, International Journal of Biometeorology 44 (2000) 198203. doi:10.1007/s004840000063.

[6] T. Lin, Y. Ho, Y. Huang, Seasonal effect of pavement on outdoor thermal environments in subtropical taiwan, Building and Environment 42 (2007) 4124-4131. doi:10.1016/j.buildenv.2006.11.031 
[7] M. Santamouris, Using cool pavements as a mitigation strategy to fight urban heat island - a review of the actual development, Renewable and Sustainable Energy Reviews 26 (2013) 224-240. doi:10.1016/j.rser. 2013.05 .047

[8] P. Pascual-Muñoz, D. Castro-Fresno, P. Serrano-Bravo, A. AlonsoEstébanez, Thermal and hydraulic analysis of multilayered asphalt pavements as active solar collectors, Applied Energy 111 (2013) 324-332. doi:10.1016/j.apenergy.2013.05.013.

[9] E. Carnielo, M. Zinzi, Optical and thermal characterisation of cool asphalts to mitigate urban temperatures and building cooling demand, Building and Environment 60 (2013) 56-65. doi:10.1016/j.buildenv.2012.11.004

[10] A. Synnefa, T. Karlessi, N. Gaitani, M. Santamouris, D. N. Assimakopoulos, C. Papakatsikas, Experimental testing of cool colored thin layer asphalt and estimation of its potential to improve the urban microclimate, Building and Environment 46 (2011) 38-44. doi:10.1016/j.buildenv.2010.06. 014.

[11] M. Pomerantz, H. Akbari, A. Chen, H. Taha, A. H. Rosenfeld, Paving materials for heat island mitigation, Ernest Orlando Lawrence Berkeley National Laboratory, 1997.

[12] H. Akbari, L. S. Rose, H. Taha, Characterizing the Fabric of the Urban Environment: A Case Study of Sacramento, California, U. S. Environmental Protection Agency, 1999.

[13] J. Gui, J. Carlson, P. E. Phelan, K. E. Kaloush, J. S. Golden, Impact of pavement thickness on surface diurnal temperatures, Journal of Green Building 2 (2007) 121-130. doi:dx.doi.org/10.3992/jgb.2.2.121.

[14] H. Akbari, A. A. Berhe, R. Levinson, S. Graveline, K. Foley, A. H. Delgado, R. M. Paroli, Aging and weathering of cool roofing membranes, in: Cool Roofing Symposium, Atlanta, GA, 2011. 
[15] A. A. Sarat, M. A. Eusuf, An experimental study on observed heating characteristics of urban pavement, Journal of Surveying, Construction and Property 3 (2012) 1-12.

[16] M. Santamouris, Using cool pavements as a mitigation strategy to fight urban heat island - a review of the actual developments, Renewable and Sustainable Energy Reviews 26 (2013) 224-240. doi:10.1016/j.rser. 2013.05.047,

[17] N. A. A. Guntor, M. F. M. Din, M. Ponraj, K. Iwao, Thermal performance of developed coating material as cool pavement material for tropical regions, Journal of Materials in Civil Engineering 26 (2014) 755-760. doi:10.1061/ (ASCE)MT . 1943-5533.0000859

[18] P. Pascual-Muñoz, D. Castro-Fresno, J. Carpio, D. Zamora-Barraza, Influence of early colour degradation of asphalt pavements on their thermal behaviour, Construction and Building Materials 65 (2014) 432-439. doi:10.1016/j.conbuildmat.2014.05.028

[19] G. Guldentops, A. M. Nejadb, C. Vuyec, W. V. den Bergh, N. Rahbara, Performance of a pavement solar energy collector: Model development and validation, Applied Energy 163 (2016) 180-189. doi:10.1016/j.apenergy. 2015.11.010.

[20] R. Mallick, B. Chen, S. Bhowmick, Harvesting energy from asphalt pavements and reducing the heat island effect, International Journal of Sustainable Engineering 2 (2009) 214-228. doi:10.1080/19397030903121950.

[21] Y. Qin, A review on the development of cool pavements to mitigate urban heat island effect, Renewable and Sustainable Energy Reviews 41 (2015) 445-459. doi:10.1016/j.rser.2015.07.177.

[22] J. Sheeba, A. Rohini, Structural and thermal analysis of asphalt solar collector using finite element method, Journal of Energy 2014 (2014) 1-9. doi:10.1155/2014/602087. 
[23] R. Mallick, B. Chen, S. Bhowmick, Harvesting heat energy from asphalt pavements: development of and comparison between numerical models and experiment, International Journal of Sustainable Engineering 5 (2012) 159169. doi:10.1080/19397038.2011.574742.

[24] A. García, M. Partl, How to transform an asphalt concrete pavement into a solar turbine, Applied Energy 119 (2014) 431-437. doi:10.1016/j. apenergy.2014.01.006.

[25] A. Chiarelli, A. García, A. Dawson, Analysis of the performance of an air-powered energy harvesting pavement, Transportation Research Record:

Journal of the Transportation Research Board 2523 (2015) 156-163. doi: $10.3141 / 2523-17$

[26] A. Chiarelli, A. García, A. Dawson, Parametric analysis of energy harvesting pavements operated by air convection, Applied Energy 154 (2015) 951-958. doi:10.1016/j.apenergy.2015.05.093.

[27] S. Kakaç, H. Liu, A. Pramuanjaroenkij, Heat Exchangers: Selection, Rating, and Thermal Design, Third Edition, CRC Press, Taylor \& Francis Group, 2012.

[28] Y. Çengel, M. Boles, Thermodynamics: An Engineering Approach, McGraw-Hill, 2010.

[29] V. Verda, S. Cosentino, S. Lo Russo, A. Sciacovelli, Second law analysis of horizontal geothermal heat pump systems, Energy and Buildings (2015) 236-240. doi:10.1016/j.enbuild.2015.09.063

[30] I. Paniagua, J. Martiín, C. Fernandez, A. Álvaro, R. Carlier, A new simple method for estimating exergy destruction in heat exchangers, Entropy 15 (2013) 474-489. doi:10.3390/e15020474

[31] G. Gan, A parametric study of trombe walls for passive cooling of buildings, Energy and Buildings 27 (1998) 37-43. doi:10.1016/S0378-7788(97) 00024-8. 
[32] WHO, Quality Assurance of Pharmaceuticals: A Compendium of Guidelines and Related Materials. Good manufacturing practices and inspection, Volume 2, World Health Organization, 2007.

[33] W. Whyte, Cleanroom Technology: Fundamentals of Design, Testing and Operation, John Wiley \& Sons, 2001.

[34] M. Dongellini, C. Naldi, G. Morini, Seasonal performance evaluation of electric air-to-water heat pump systems, Applied Thermal Engineering 90 (2015) 1072-1081. doi:10.1016/j. applthermaleng. 2015.03.026.

[35] C. Gommes, A. Bons, S. Blacher, J. Dunsmuir, A. Tsou, Practical methods for measuring the tortuosity of porous materials from binary or gray-tone tomographic reconstructions, American Institute of Chemical Engineers 55 (2009) 2000-2012. doi:10.1002/aic.11812.

[36] A. Bejan, Convection Heat Transfer, Wiley, 2013.

[37] M. Kutay, A. Aydilek, E. Masad, Estimating directional permeability of hot-mix asphalt by numerical simulation of microscale water flow, Transportation Research Record: Journal of the Transportation Research Board (2001) 29-36doi:10.3141/2001-04 